\title{
Research and Development for Technology Evolution Potential Forecasting System
}

\author{
Changqing Gao, Shukun Cao, Yuzeng Wang, Changsheng Ai, and Xiangbo Ze \\ School of Mechanical Engineering, University of Jinan, 250022 Jinan, China \\ chq_gao@hotmail.com
}

\begin{abstract}
Technology forecasting is a powerful weapon for many enterprises to gain an animate future. Evolutionary potential radar plot is a necessary step of some valuable methods to help the technology managers with right technical strategy. A software system for Technology Evolution Potential Forecasting (TEPF) with automatic radar plot drawing is introduced in this paper. The framework of the system and the date structure describing the concrete evolution pattern are illustrated in details. And the algorithm for radar plot drawing is researched. It is proved that the TEPF system is an effective tool during the technology strategy analyzing process with a referenced case study.
\end{abstract}

Keywords: Technology Forecasting, TRIZ, Innovation Design, Evolution Potential.

\section{Introduction}

Technology advancement is the core power for economic development. Technology forecasting is the activity to predict the future of technology. And it can help the enterprises to develop tomorrow' products in the current times. In order to gain the advantage over their competitors and dominate the market, technology forecasting is the key factors for the animate future of each technology-oriented company [1]. TRIZ (the Russian acronym for the theory) is the knowledge-based, systematic approach to innovation. Developed in the former Soviet Union by Genrich S. Altshuller (1926-1998) and his school, TRIZ methods are drawn from analysis of the most innovative inventions in different industries, technologies, and fields of engineering. TRIZ confirms that the evolution of a technical system is not a random process. It is governed by certain objective laws. TRIZ is composed of the rules that can guide the inventive activities [2-3]. Evolution theory is an effective tool of TRIZ to practice technology forecasting and technology strategy analysis. It does not just predict the trend of technology development, it also exhibit the corresponding possible structure states for new product development. TRIZ evolution theory is gradually applied in many industry fields, such as cutting technology, telephone and crane [4-6]. During the process of the development opportunities analysis of product structure, Evolution Potential Radar Plot is an indispensable picture for decision-making. The picture drawing is usually a time-taking process, during which the plentiful information from the different mediums should be 
collected together, such as the different evolution patterns and the corresponding cases. So the professional software for technology forecasting supporting automatic Evolution Potential Radar Plot drawing is a smart tool for the new product development.

\section{TRIZ Evolution Theory}

The researching analysis indicates that the evolution process of an engineering system is ruled by some certain patterns. TRIZ considers every significant improvement of any design or engineering system to be a step in its evolution. The most important steps in the development of design and engineering systems in different fields and industries are common. They are called the Laws of Engineering System Evolution, which are based on analysis of thousands of patents and improvement of thousands of products and technologies. According to the Laws of Engineering System Evolution, the S-curve of any product has a typical life cycle of pregnancy, birth, childhood, adolescence, maturity and decline. A brand new design with competitive advantage can be obtained by the Laws of Engineering System Evolution, which always means great improvement over the current design [7].

\subsection{Evolution Patterns}

Accompanied by the development of TRIZ methodology, there appeared some different system for evolution patterns. According to reference [8-10], Altshuller, Zusman and Darrell represented ten kinds of evolution patterns, eight kinds of evolution patterns and eleven kinds of evolution patterns respectively.

Those evolution patterns are some generic technology evolution trends which determine the evolution of technical systems. The eleven patterns of evolution for the engineering system are listed in Table 1.

Table 1. Eleven Patterns of Evolution

\section{Patterns of Evolution}

1. Evolution towards increased Ideality

2. increased dynamic state within systems

3. increased system segmentation

4. increased space segmentation

5. increased surface segmentation

6. increased controllability

7. increased complexity followed by reduced complexity

8. use of all available physical dimensions within a system

9. decreased number of energy conversions

10. increased rhythm co-ordination

11. increased action co-ordination 


\subsection{Evolution Potential}

All the technical systems evolve in the direction of increasing ideality. IFR (Ideal Final Result) is the evolutionary limit for them. Evolution theory can help us forecast the evolution trends by evolution patterns or routes. During this approach, the important thing we should know is how much close the technology system has evolved to its ideality. Evolution Potential Radar Plot is just the tool to reach that target, as shown in Figure 1. In Figure 1, each radial with an arrow represents one of the TRIZ evolution patterns. Those existed evolution pattern is the one which has been selected to help us for technology forecasting. And the serial number of each evolution pattern according to Table 1 is marked near the end of the radial. The outside boundary of the

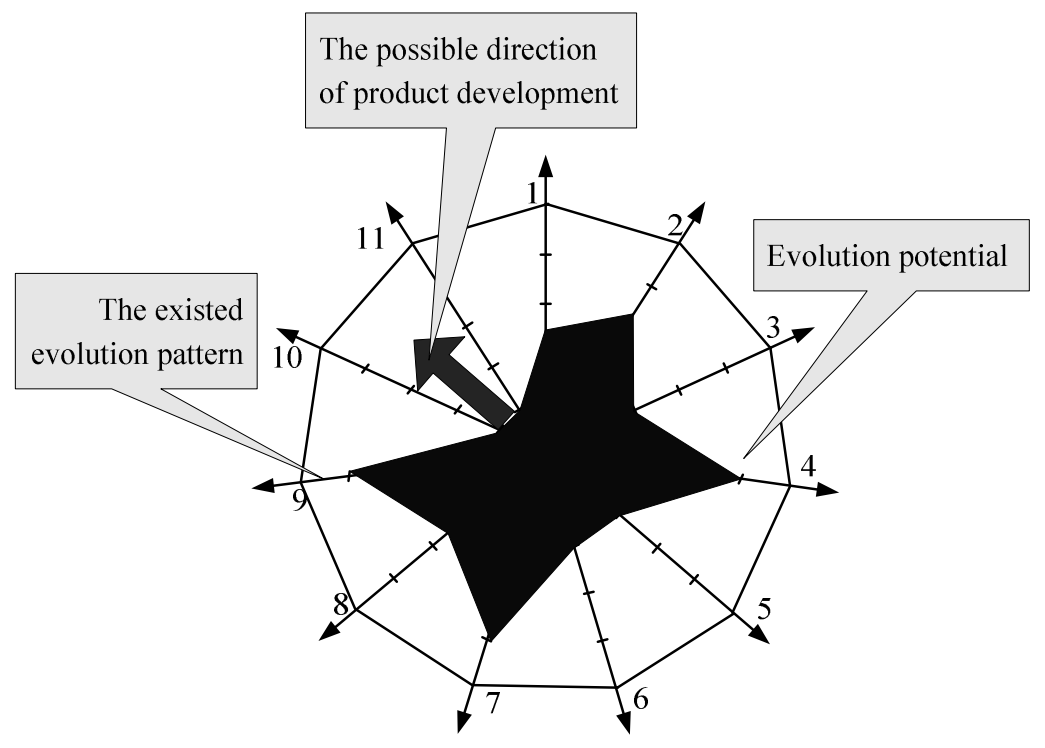

Fig. 1. Evolution Potential Radar Plot

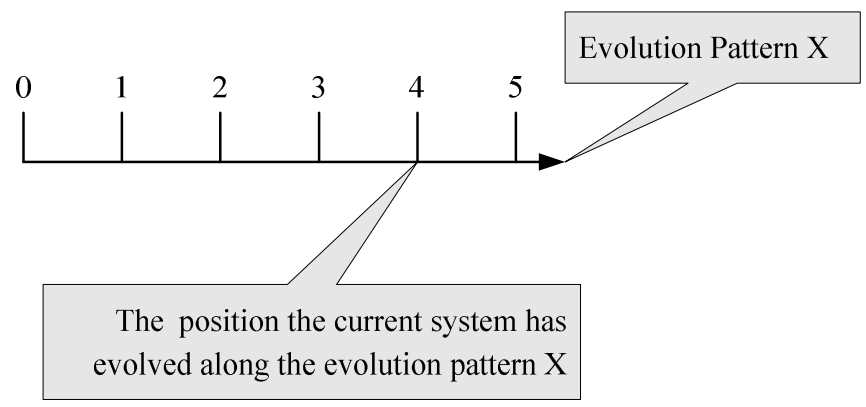

Fig. 2. Position a system in relation to the evolution patterns 
Plot represents the corresponding evolution limit. Each radial is divided into five sections, by which the position the current system has evolved along the evolution pattern can be valued, as shown in Figure 2. The meaning of the positions is listed in Table 2. The shaded area represents how far the current system has evolved. The area between shaded area and the boundary represents the evolution potential [11].

Table 2. Position a system in relation to the evolution patterns

\begin{tabular}{cl}
\hline Position Value & \multicolumn{1}{c}{ The meaning of the Value } \\
0 & $\begin{array}{l}\text { Product hasn't evolved along this pattern. It's necessary to } \\
\text { develop product along this pattern. } \\
\text { Product just began its evolution along this pattern. A further more } \\
\text { development is possible. } \\
3\end{array}$ \\
5 & $\begin{array}{l}\text { Product has evolved by this pattern with a medium-past degree. } \\
\text { Product has reached its evolution limit. It is not necessary to } \\
2,4\end{array}$ \\
& $\begin{array}{l}2 \text { represent the state between position } 1 \text { and } 3.4 \text { represent the state } \\
\text { between position } 3 \text { and 5. }\end{array}$
\end{tabular}

\section{Technology Evolution Potential Forecasting System}

To develop a Technology Evolution Potential Forecasting (TEPT) System to ease the process of producing Evolution Potential Radar Plot is significant for technology strategy analyzing. Users can exchange information with knowledge base of the system. Consequentially, Evolution Potential Radar Plot can be output automatically. The frame of the system is shown in Figure 3. TEPT System is implemented with Microsoft Visual Basic 6.0 program tool under Windows XP surroundings.

\subsection{Date Structure for Evolution Pattern Radial}

In order to represent the Evolution Pattern information in computer system, the key task is to establish a date structure for Evolution Pattern describing. As shown in Figure 1, each radial with an arrow represents one of the TRIZ evolution patterns. A list is founded to complete this task. As shown in Figure 4, ER is a list for describing a Evolution Pattern Radial with Five logic segments. 'Sn' represents the serial number of Evolution Patterns. ' $\mathrm{Na}$ ' is the name of evolution pattern or route. 'Cs' notes the information recording the current position the system has evolved along the evolution pattern $\mathrm{X}$. ' $\mathrm{Hi}$ ' is the highest position value of evolution. And this value is evaluated as 5 in this system according to Table 2 . 'In' is the index of evolutionary case. 


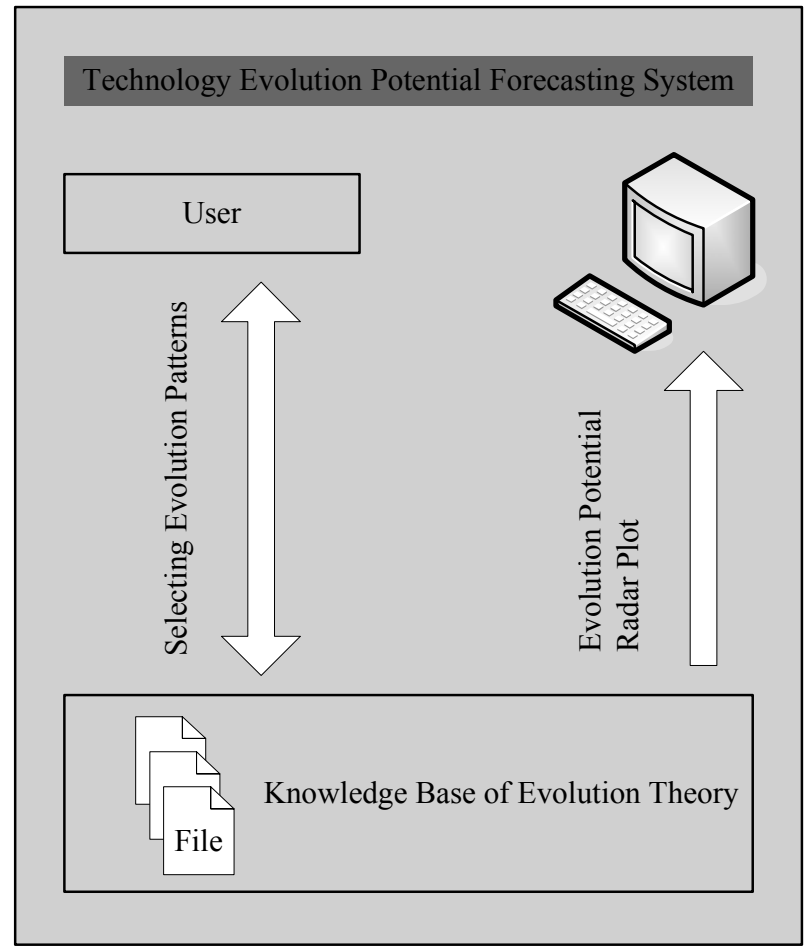

Fig. 3. Technology Evolution Potential Forecasting System

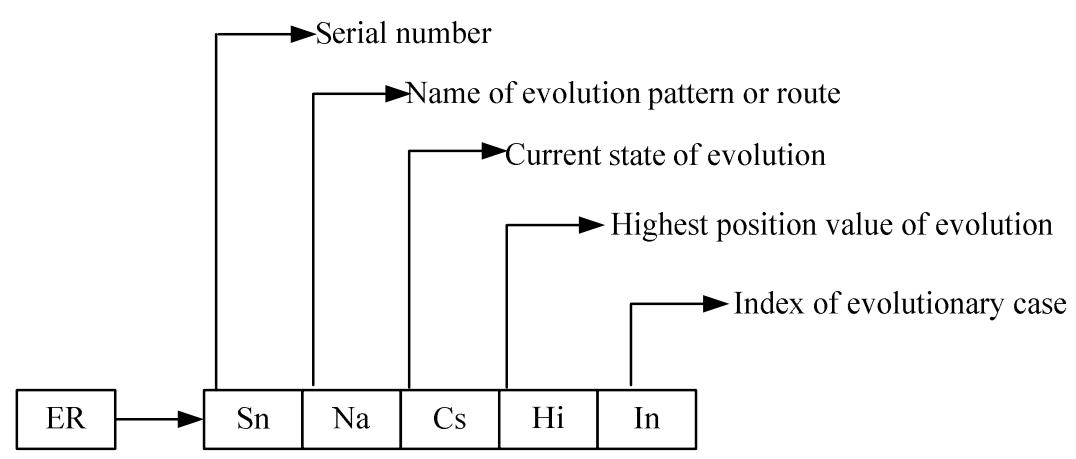

Fig. 4. Date structure for Evolution Pattern Radial

\subsection{Geometrical Relationship of Evolution Pattern Radial}

There are some radials in the Evolution Pattern Radar Plot with serial numbers. The angle between radial ' $i$ ' and radial ' $i+1$ ' can be noted by $\theta$. In Evolution Pattern Radar Plot, is a constant, which depends on the amount of the radials. If there are totally $\mathrm{N}$ radials representing $\mathrm{N}$ Evolution Patterns, then angle $\theta$ can be represented by formula (1) listed below. 


$$
\theta=\frac{2 \times P I}{N} \quad i=1,2, \cdots, N
$$

The first radial is laid on horizontal level with the direction of positive $\mathrm{X}$.

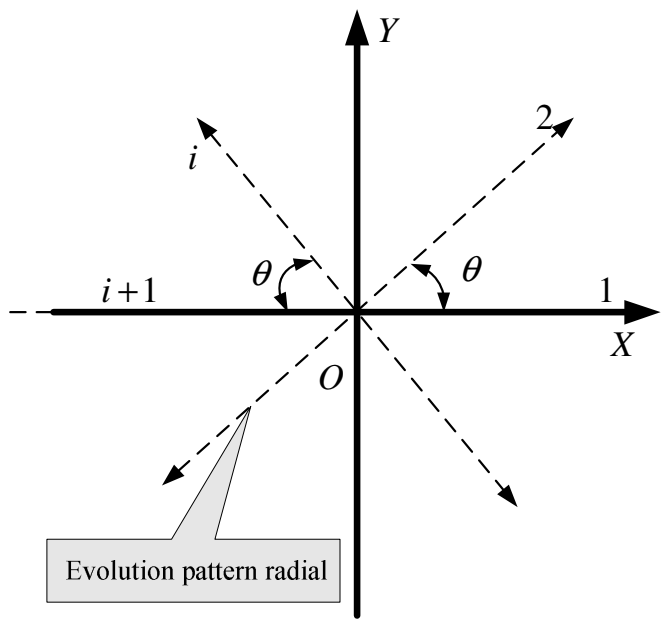

Fig. 5. Evolution Pattern Radial in a plane with XY dimensionality

\subsection{Coordinate Conversion of Picture Area}

Control Object can be used to implement the drawing work. Under Visual Basic Programming surroundings, PictureBox can do this job. As shown in Figure 6, there is a PictureBox area with rectangle shape. Width and Height of the area are noted above. Before the Evolution Potential Radar Plot drawing, coordinate conversion between computer space and user space should be done. The computer space is usually located by coordinate system XOY, as shown in the LeftTop corner of the rectangle area. $\mathrm{X}^{\prime} \mathrm{O}^{\prime} \mathrm{Y}^{\prime}$ is used as the coordinate system for the user space because of our habit. The origin of X'O'Y' system can be positioned in the computer space by formula (2).

$$
\left\{\begin{array}{l}
O^{\prime}\left(X^{\prime}\right)=\frac{\text { Width }}{2} \\
O^{\prime}\left(Y^{\prime}\right)=\frac{\text { Height }}{2}
\end{array}\right.
$$

There are N radials in user space. Each radial's start point is superposed on the origin of X'O'Y' system. And each radial forms an angle with positive X' axis, which is noted by ' $\alpha$ '. According to formula (1), $\alpha(i)$, which is formed by the ith radial, can be calculated by formula (3).

$$
\alpha(i)=(i-1) \times \theta \quad i=1,2, \cdots, N
$$




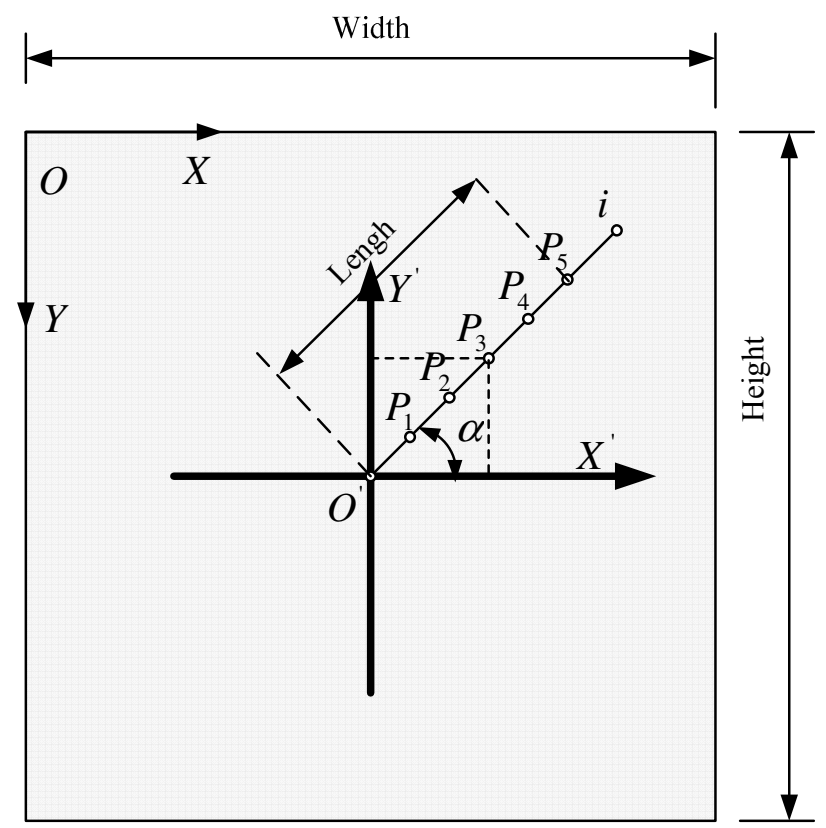

Fig. 6. Conversion between coordinate system XOY and X'O'Y'

Each radial is divided into 5 sections equally. The corresponding positions that are used to represent the state the current technical system has reached on the evolution pattern are noted by $P_{s}(1 \leq s \leq 5)$. Then the $P_{s}$ on the ith radial can be located by its X and Coordinates. As shown in Figure 6, the Coordinates can be represented by formula (4).

$$
\left\{\begin{array}{l}
P_{s i}\left(X^{\prime}\right)=\frac{\text { Width }}{2}+\left(s \times \frac{\text { Lengh }}{5}\right) \times \cos (\alpha(i)) \\
P_{s i}\left(Y^{\prime}\right)=\frac{\text { Height }}{2}-\left(s \times \frac{\text { Lengh }}{5}\right) \times \sin (\alpha(i))
\end{array} \quad 1 \leq s \leq 5 \text { And } i=1,2, \cdots, N\right.
$$

Length can be identified in advance. According to the formulas, the coordinates of all the special positions on each radial can be calculated. Then the Evolution Potential Radar Plot can be lined based on the coordinates. The results are exampled by Figure 7.

\subsection{Knowledge Base of Evolution Theory}

The concrete evolution pattern or route with corresponding examples can be recorded in a special file with some literal explanations. They are the base for the special cases. And the cases can be showed in the TEPF System software by the index of them, as shown in Figure 8. 

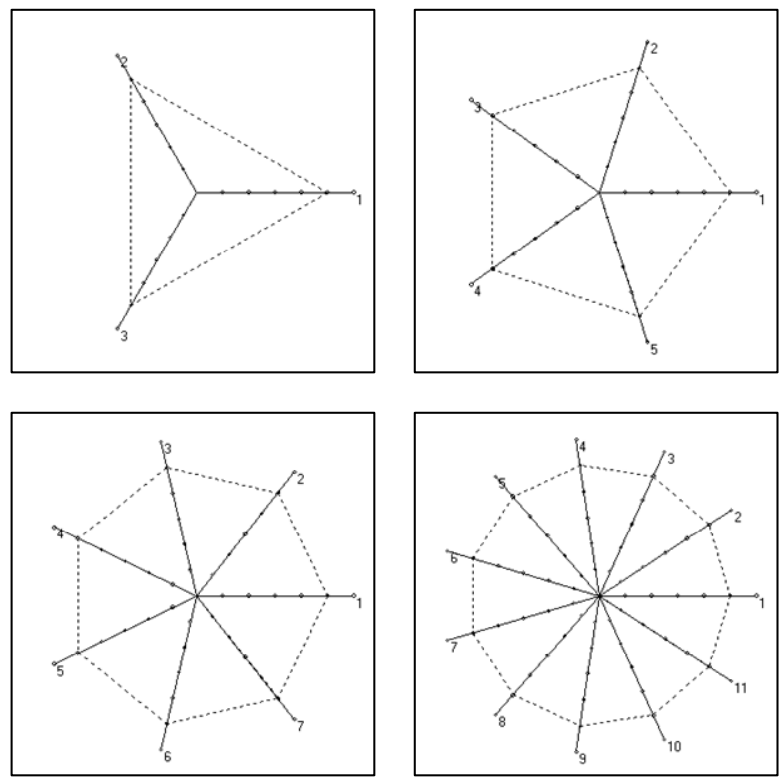

Fig. 7. Unfinished Evolution Potential Radar Plot

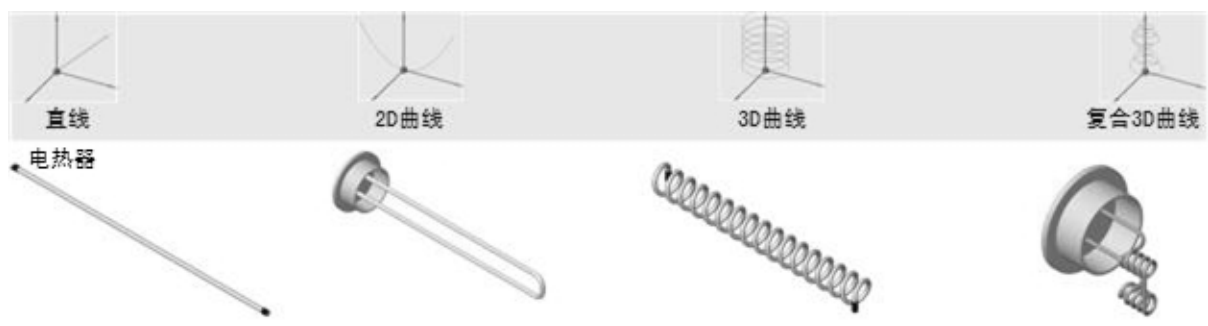

Fig. 8. Evolution pattern or route with examples

\section{Case Study}

The TEPF System with Chinese version is developed to ease the process of evolution potential forecasting for the technical systems. TEPF System provide the functions including selecting evolution pattern, indexing the corresponding cases, positioning a system in relation to the evolution patterns and Radar Plot drawing, etc.

According to reference [4] authored by Zhang Fuying, the development on cutting technology based on TRIZ Directed Evolution was discussed in details. As shown in Figure 9, the analyses of the reference [4] for Evolution Potential Forecasting and Radar Plot drawing can be done easily by TEPF System. It is quite effective for technical strategy analyses and TRIZ education and training. 


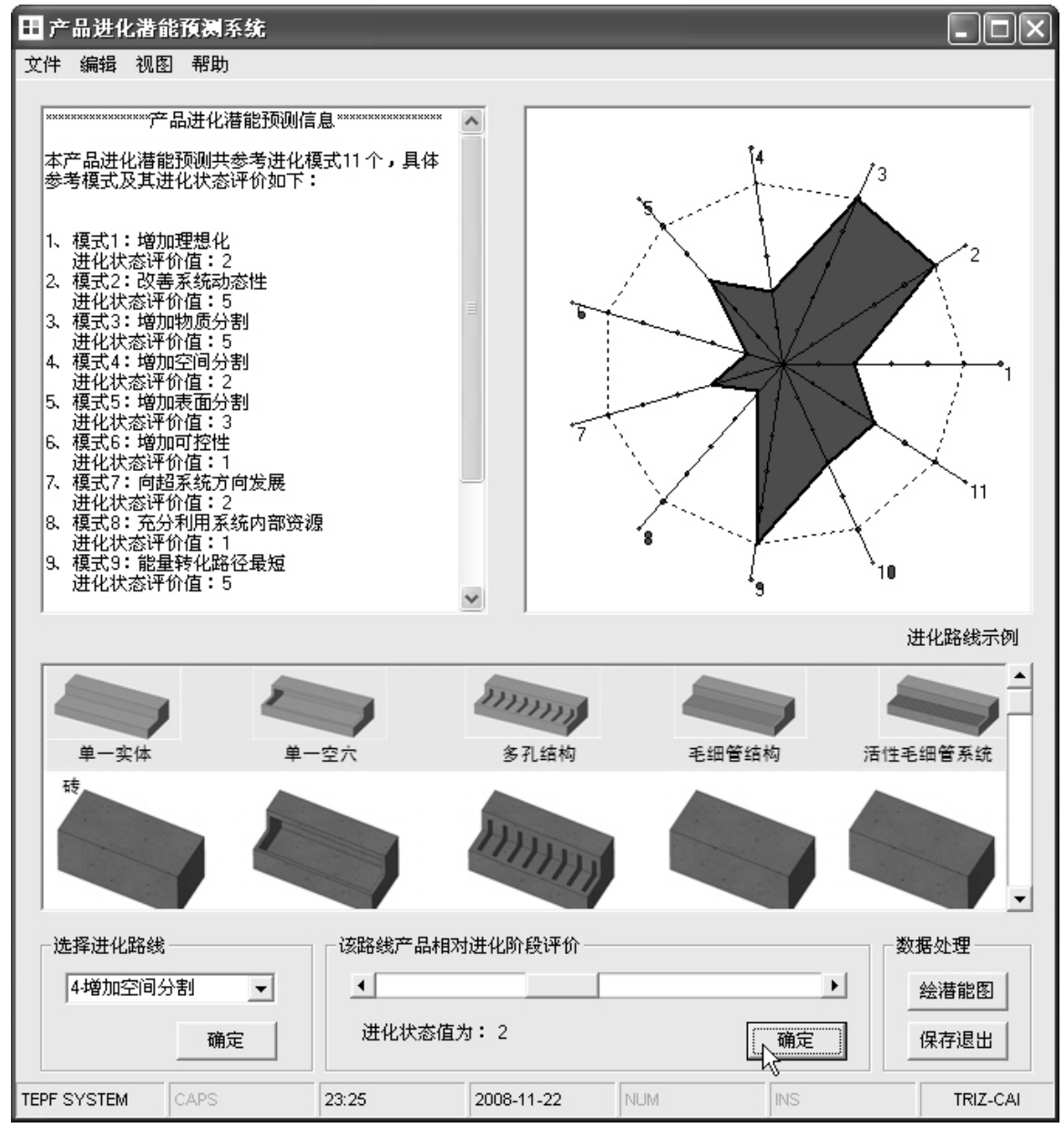

Fig. 9. Interface of TEPF System

\section{Conclusions}

With the referenced case study, it is proved that TEPF System is a smart tool during the technology strategy analyzing process and TRIZ education. It lessens the labor intension of technology managers and improves the efficiency of the technology forecasting process. And there are some further research works that should be carried on in the near future, including extending the knowledge base for evolution patterns and the management of forecasting information.

\section{Acknowledgements}

This research is sponsored by National Key Project of Scientific and Technical Supporting Programs Funded by Ministry of Science \& Technology of China during the 11th 
Five-year Plan (NO. 2006BAF02A24, Doctor Research Fund from University of Jinan (Grant No. B0625) and Key Subject Fund from Shandong Province (Grant No. XTD0713).

\section{References}

1. Victor, R.F., Eugene, I.R.: Guided technology evolution (TRIZ technology forecasting) (January 1999), http: / /www . triz-journal. com

2. Savransky, S.D.: Engineering of creativity. CSC Press, New York (2000)

3. Tan, R.H.: Theory of inventive problem solving, August 15-18. Science Press, Beijing (2004)

4. Zhang, F., Xu, Y., Ping, W.: Development on cutting technology based on TRIZ Directed Evolution. Journal of Nanjing University of Aeronautics \& Astronautics 37, 190-193

5. Zhao, X., Ying, Z.: Application of TRIZ Evolution Theory: Analyzing the Formal Evolution of Telephone. Modern Art and Design (167), 108-109 (2007)

6. Li, H., Zhang, M., Han, L.-f., et al.: Directed evolution theory of TRIZ based crane technology forecasting. Journal of Shandong Jianzhu University 22(3), 268-282 (2007)

7. Terninko, J., Zusman, A., Zlotin, B.: Systematic innovation. St. Lucie Press, New York (1998)

8. Altshuller, G.S.: Creativity as an exact science. Gordon \& Breach, New York (1984)

9. Zusman, A., Zlotin, B., Zsiniev, G.: An application of directed evolution, http://www.ideationtriz.com/Endoscopic_Case_Study.htm

10. Darrell, M.: Evolutionary-potentialTM in technical and business systems [DB/OL] (June 2002), http: / / www.triz-journal.com

11. Zhang, F., Zhang, L., Wang, P.: Study on Product Innovative Design Based on TRIZ Evolution Theory. Transactions of Chinese Society for Agricultural Machinery 39(2), 116-119 (2008) 